Editorial

\title{
Particle Production in Small and Large Systems at High-Energy and High-Density Frontiers
}

\author{
Fu-Hu Liu $(\mathbb{D}),{ }^{1}$ Sakina Fakhraddin $\mathbb{D}^{2},{ }^{2,3}$ Raghunath Sahoo ${ }^{4}{ }^{4}$ \\ Edward K. Sarkisyan-Grinbaum, ${ }^{5,6}$ and Bhartendu K. Singh ${ }^{7}$ \\ ${ }^{1}$ Institute of Theoretical Physics, Shanxi University, Taiyuan 030006, China \\ ${ }^{2}$ Physics Department, College of Sciences \& Arts in Riyadh Alkhabra, Qassim University, Saudi Arabia \\ ${ }^{3}$ Physics Department, Faculty of Science, Sana'a University, P.O. Box 13499, Sana'a, Yemen \\ ${ }^{4}$ Department of Physics, Indian Institute of Technology Indore, Indore 453552, India \\ ${ }^{5}$ Experimental Physics Department, CERN, 1211 Geneva 23, Switzerland \\ ${ }^{6}$ Department of Physics, The University of Texas at Arlington, Arlington, TX 76019, USA \\ ${ }^{7}$ Physics Department, Banaras Hindu University, Varanasi 221005, India \\ Correspondence should be addressed to Fu-Hu Liu; fuhuliu@163.com
}

Received 28 July 2021; Accepted 28 July 2021; Published 15 August 2021

Copyright (c) $2021 \mathrm{Fu}-\mathrm{Hu}$ Liu et al. This is an open access article distributed under the Creative Commons Attribution License, which permits unrestricted use, distribution, and reproduction in any medium, provided the original work is properly cited.

Particle production in small systems (proton-proton and proton-nucleus collisions) and in large systems (nucleusnucleus collisions) at high-energy and high-density frontiers displays both similar and different behaviors. Comparing this behavior is very useful for researchers to study the properties of particle production and system evolutions. These properties are related to the signatures of the formation of a new state of matter, quark-gluon plasma (QGP), which is predicated by quantum chromodynamics (QCD). These properties are also related to the mechanisms of particle production, which are inevitably appearing in collisions.

At the high-energy frontier, the Relativistic Heavy Ion Collider (RHIC) in the Brookhaven National Laboratory (BNL) and the Large Hadron Collider (LHC) in the European Organization for Nuclear Research (CERN) provide abundant data produced in small and large systems. These data include, but are not limited to, transverse momentum spectra, anisotropic flows, statistical or dynamical fluctuations of light flavor particles, heavy flavor particles, jets, and leptons. It is expected that the effect of cold nuclei or medium affects particle production in large systems, compared with small systems.

At the high-density frontier, the beam energy scan (BES) program and its second round (BESII) and fixed target exper- iments at the RHIC, the BES at the Super Proton Synchrotron (SPS) in CERN, the Facility for Antiproton and Ion Research (FAIR) in Germany Center for Heavy Ion Research (GSI), and others perform nucleus-nucleus collisions at high baryon number densities around the expected critical point of hadronic matter transition to QGP. It is expected that the spectra of particles are different in small and large systems at the high-density and high-energy frontiers.

In small and large systems at high-energy and highdensity frontiers, the features of particle production and system evolution attract high interest and are extensively studied both experimentally and theoretically. It is believed that the properties of particle production and system evolution in small and large systems at high-energy and high-density frontiers are different. These properties are related to those of the early universe considered to go through the stages of high density and high temperature.

This special issue concerns many topics on particle production in small and large systems at high-energy and high-density frontiers, for example, (i) description of particle distributions and correlations and studies of statistical laws and dynamical properties of particle production; (ii) extraction of different quantities based on the particle spectra, correlations, and yield ratios; (iii) studies of the properties of 
particle production and system evolution in small and large systems at the high-energy frontier; (iv) studies of the properties of particle production and system evolution in small and large systems at the high-density frontier; and (v) comparison of the properties of particle production and system evolution in small and large systems at high-energy and high-density frontiers.

In the article "Event Shape and Multiplicity Dependence of Freeze-Out Scenario and System Thermodynamics in Proton+Proton Collisions at $\sqrt{s}=13 \mathrm{TeV}$ Using PYTHIA8" by $\mathrm{S}$. Tripathy et al., the authors have studied the recent observations of QGP-like conditions in high-multiplicity proton +proton ( $\mathrm{pp}$ ) collisions from the ALICE experiment at the LHC and warranted an introspection whether to use pp collisions as a baseline measurement to characterize heavy-ion collisions for the possible formation of a QGP. This article has shown that a double differential study of the particle spectra and thermodynamics of the produced system as a function of charged particle multiplicity and transverse sphericity in pp collisions would shed light on the underlying event dynamics. Transverse sphericity, one of the event shape observables, allows separating the events in terms of jetty and isotropic events. The authors analyzed the identified particle transverse momentum spectra as a function of charged particle multiplicity and transverse sphericity using Tsallis nonextensive statistics and the Boltzmann-Gibbs Blast-Wave (BGBW) model in pp collisions using the PYTHIA8 event generator. The extracted parameters such as temperature, radial flow, and nonextensive parameters are shown as a function of charged particle multiplicity for different sphericity classes. The authors observed that the isotropic events approach thermal equilibrium while the jetty ones remain far from equilibrium. Meanwhile, the authors argued that, while studying the QGP-like conditions in small systems, one should separate the isotropic events from the sphericity-integrated events, as the production dynamics are different.

In the article "Study of Production of (Anti-)deuteron Observed in $\mathrm{Au}+\mathrm{Au}$ Collisions at $\sqrt{s_{\mathrm{NN}}}=14.5,62.4$, and $200 \mathrm{GeV}$ " by Y. Yuan, the author has studied the transverse momentum distributions of deuterons and anti-deuterons in $\mathrm{Au}+\mathrm{Au}$ collisions at $\sqrt{s_{\mathrm{NN}}}=14.5,62.4$, and $200 \mathrm{GeV}$ with different centralities $(0-10 \%, 10-20 \%, 20-40 \%, 40-60 \%$, and $60-80 \%)$ in the framework of the multisource thermal model. The results show that the transverse momentum spectra are conformably and approximately described by the Tsallis distribution which is from the Tsallis nonextensive statistics and has the form satisfying the thermodynamic consistency. The dependences of the derived quantity (average transverse momenta) and the two free parameters (effective temperature and entropy index) on event centrality are obtained. It is found that the effective temperature extracted from this study increases with the decrease of centrality percentage at the same incident energy, and the entropy index decreases with the decrease of centrality percentage at the same incident energy. At the same collision centrality, the two parameters increase with the increase of incident energy. However, the kinetic freeze-out temperature and the evolution of time during the collision have yet to be studied in depth.
In the article "Calculation of Binding Energy and Wave Function for Exotic Hidden-Charm Pentaquark" by F. Chezani Sharahi and M. Monemzadeh, the authors have studied the pentaquark $P_{c}(4380)$ system consisting of baryon $\sum_{c}$ and $\bar{D}^{*}$ meson. The obtained potential for the pentaquark in the literature was presented and expanded. In this study, the expanded potential was replaced with the Schrödinger equation, and that was solved as a bound state of two-body systems. By solving this to analytically approach and according to the values of constants and cutoff, 10 nonlinear differential equations and binding energy $E_{B}$ of pentaquark $P_{c}$ and wave function coefficients were obtained. The resulting $E_{B}$ and wave function, which are presented in the form of tables and diagrams in this study, could confirm the existence of a bound state of pentaquark $P_{c}(4380)$. It is specified that the wave function plots tend to be zero at a given value. The maximum radius of pentaquark $P_{c}$ was found out in the range from 23.67 to $25.64 \mathrm{fm}$. The authors observed that the calculated values match with the findings of others regarding the mass of $P_{c}(4380)$. The advantage of this study is in the methodology. In fact, other references are numerically calculated, and this study delivers the results analytically. The results obtained in this study are more comprehensive and complete compared to others.

The article "Transverse Momentum and Pseudorapidity Spectrum of the Top Quark, Lepton, and $b$ Jet in ProtonProton Collisions at LHC" by L.-N. Gao and E.-Q. Wang has studied the transverse momentum and pseudorapidity spectra of the top quarks and their decay products, the $t \bar{t}$ system, and the total number of jets in proton-proton collisions at 7,8 , and $13 \mathrm{TeV}$ by using the Tsallis-Pareto-type function and the three-source Landau hydrodynamic model, respectively. The related parameters, such as the effective temperature of the interacting system, the nonextensivity of the process, and the width of the pseudorapidity distribution, are extracted. This study shows that for the products, top quarks, leptons, and $b$ jets, the width of the pseudorapidity distribution shows an increasing trend with the collision energy. The effective temperature of the interacting system shows a slight increase with the collision energy; this phenomenon may be affected by QGP. Based on the related parameters, the values of average momentum and initial temperature are calculated. Both values are very large; this could mean the high excitation degree of the interesting system.

The article "Centrality Dependence of Chemical FreezeOut Parameters and Strangeness Equilibration in RHIC and LHC Energies" by D. Biswas has studied the centrality variation of chemical freeze-out parameters from yield data at midrapidity of charged pions, charged kaons, protons, and antiprotons for collision energies of RHIC, RHIC-BES, and LHC. The author has considered a simple hadron resonance gas model and employed a formalism involving conserved charges $(B, Q, S)$ of QCD for parameterization. Along with temperature and three chemical potentials $\left(T, \mu_{B}, \mu_{Q}\right.$, and $\left.\mu_{S}\right)$, a strangeness undersaturation factor $\left(\gamma_{S}\right)$ has been used to incorporate the partial equilibration in the strange sector. The obtained freeze-out temperature does not vary much with centrality, whereas the chemical potentials and $\gamma_{S}$ seem to have a significant dependence. The strange hadrons are 
found to deviate from a complete chemical equilibrium at freeze-out at peripheral collisions. This deviation appears to be more prominent as the collision energy decreases at lower RHIC-BES energies. The author has also shown that this departure from equilibrium reduces towards central collisions, and strange particle equilibration may happen after a threshold number of participants in large collision systems. These results can be compared with those from thermal and kinetic freeze-out.

This issue brings together a collection of articles on particle production in small and large systems at high-energy and high-density frontiers. We hope this will be a useful issue for researchers working in related areas. Meanwhile, we regret that more manuscripts submitted for publication in this issue have not been accepted following the reviewer's reports.

\section{Conflicts of Interest}

The editors declare that they have no conflicts of interest regarding the publication of this special issue.

\section{Acknowledgments}

We sincerely thank all authors and reviewers of this special issue.

$\mathrm{Fu}-\mathrm{Hu} \mathrm{Liu}$

Sakina Fakhraddin

Raghunath Sahoo

Edward K. Sarkisyan-Grinbaum

Bhartendu K. Singh 